\title{
Pulmonary alveolar proteinosis: diagnosis using routinely processed smears of bronchoalveolar lavage fluid
}

\author{
Tetuo Mikami, Yoshiko Yamamoto, Masaru Yokoyama, Isao Okayasu
}

Department of Pathology, School of Medicine, Kitasato

University,

Sagamihara, Kanagawa, Japan

T Mikami

I Okayasu

Department of Pathology, Tokyo

Medical and Dental

University Hospital,

Tokyo, Japan

Y Yamamoto

Cytology Section, Department of Pathology, Kitasato University Hospital M Yokoyama

Correspondence to: Dr Mikami, Department of Pathology, School of Medicine, Kitasato University, 1-15-1, Kitasato, Sagamihara, Kanagawa, 228 , Japan.

Accepted for publication 10 October 1997

\begin{abstract}
Aims-For the diagnosis of pulmonary alveolar proteinosis from bronchoalveolar lavage specimens it is normally necessary to make an ultrastructural examination. However, this is thought to be impractical for bronchoalveolar lavage specimens that have been routinely fixed in ethanol. In the present study, bronchoalveolar lavage cytology smears on slide glasses were examined directly ultrastructurally to make a diagnosis of pulmonary alveolar proteinosis.

Methods-Bronchoalveolar lavage smears from three pulmonary alveolar proteinosis patients were stained with Papanicolaou and periodic acid-Schiff (PAS) for identification of amorphous globular structures. Subsequently, they were refixed with glutaraldehyde and osmium tetroxide, and embedded in epoxy resin. Ultrathin sections were cut and examined ultrastructurally.

Results-Papanicolaou stained specimens from pulmonary alveolar proteinosis patients contained scattered amorphous or granular globules, $20-50 \mu \mathrm{m}$ in diameter, which were PAS positive. Ultrastructural examination of the globules revealed multilamellated structures, characteristic of pulmonary alveolar proteinosis, in all cases.

Conclusions-In general, it is thought that the morphological diagnosis of pulmonary alveolar proteinosis from bronchoalveolar lavage specimens requires both cytological and ultrastructural examination. However, the amorphous globules evident on cytology smears proved to contain multilamellated structures so that they can themselves be used as diagnostic evidence.

(F Clin Pathol 1997;50:981-984)
\end{abstract}

Keywords: pulmonary alveolar proteinosis; bronchoalveolar lavage; multilamellated structure

Pulmonary alveolar proteinosis is a rare disease first described by Rosen et al in $1958 .^{1}$ It is characterised by deposition within the air spaces of a granular extracellular material composed of proteins and lipids that is periodic acid-Schiff (PAS) positive and diastase resistant. ${ }^{23}$ Its diagnosis is based mainly on histological findings obtained with transbronchial lung biopsy or open lung biopsy specimens. However, transbronchial lung biopsy specimens are often so small that they do not provide specific evidence. Because the characteristic intra-alveolar material is localised mainly in the pulmonary hilar region, ${ }^{2}$ open lung biopsy may also fail to ensure diagnosis. Bronchoalveolar lavage can sample a much wider area and is safer for the patient than transbronchial lung biopsy and/or open lung biopsy.

Pulmonary alveolar proteinosis cytology findings from bronchoalveolar lavage specimens have been reported previously, ${ }^{4-7}$ but results of Papanicolaou staining are not considered sufficient for a definitive diagnosis and it is generally thought necessary to confirm the presence of characteristic multilamellated structures under the electron microscope. ${ }^{467}$ However, it is not practical to process all routine bronchoalveolar lavage specimens for ultrastructural examination. Therefore, in the present study we directly compared the ultrastructure of cytology materials prepared initially for routine Papanicolaou smears. Our purpose was to prove that the globules seen under the light microscope have characteristic electron microscopic features, and thus to assess the possibility of pulmonary alveolar proteinosis diagnosis from routinely processed bronchoalveolar lavage smears alone.

\section{Methods}

Three cases of pulmonary alveolar proteinosis (including idiopathic and secondary types) were collected by reviewing the pathology reports of Kitasato University Hospital and the Tokyo Medical and Dental University Hospital. In all three cases, transbronchial lung biopsy was performed to prove the presence of PAS positive and diastase digestion resistant intra-alveolar material. Clinical information on the cases is summarised in table 1. 
Table 1 Clinical details of the three patients

\begin{tabular}{|c|c|c|c|c|}
\hline $\begin{array}{l}\text { Case nol } \\
\text { sex }\end{array}$ & $\begin{array}{l}\text { Age } \\
\text { (years) }\end{array}$ & Symptoms/signs & Background disease & $\begin{array}{l}\text { Final diagnostic } \\
\text { procedure }\end{array}$ \\
\hline $1 / \mathrm{F}$ & 47 & Dyspnoea & $\begin{array}{l}\text { Chronic myelogenous } \\
\text { leukaemia }\end{array}$ & $\begin{array}{l}\text { Transbronchial lung } \\
\text { biopsy }\end{array}$ \\
\hline $2 / F$ & 50 & Fever, coughing & None (idiopathic) & $\begin{array}{l}\text { Transbronchial lung } \\
\text { biopsy }\end{array}$ \\
\hline $3 / \mathrm{M}$ & 33 & $\begin{array}{l}\text { Abnormal shadow } \\
\text { on chest } x \text { ray }\end{array}$ & None (idiopathic) & $\begin{array}{l}\text { Transbronchial lung } \\
\text { biopsy }\end{array}$ \\
\hline
\end{tabular}

Smears of bronchoalveolar lavage fluid sediment from the patients were fixed routinely with $95 \%$ ethanol and subjected to Papanicolaou staining. After examination under the light microscope, coverslips were removed by rinsing in xylene. Following decoloration by rehydration, PAS staining was carried out. The slides were examined microscopically and used for electron microscopy as described below.

The smears were fixed with $2.5 \%$ glutaraldehyde for 30 minutes, rehydrated with graded concentrations of ethanol, and postfixed with $1 \%$ osmium tetroxide for one hour. The smears were then dehydrated in graded ethanols up to $100 \%$ and embedded by inverting a gelatin capsule of fresh epoxy resin over each slide. The epoxy resin was polymerised by overnight incubation at $37^{\circ} \mathrm{C}$ and a further incubation at $60^{\circ} \mathrm{C}$ for $24-36$ hours. The epoxy resin blocks were removed from the slides by heating and ultrathin sections were cut. These were stained with $3.5 \%$ uranyl acetate and Reynolds' lead citrate and examined with a transmission electron microscope (H-600; Hitachi, Tokyo, Japan).

To serve as controls, Papanicolaou stained routine cytology smears from bronchoalveolar lavage fluid samples of 31 patients without pul- monary alveolar proteinosis (initially diagnosed as "no malignant cells") were reviewed. These control patients consisted of 20 men and 11 women aged between 39 and 82 years old. The clinical diagnoses before bronchoalveolar lavage were as follows: 15 cases of suspected lung tumour, three cases of suspected pulmonary haemorrhage, three cases of pneumonia, two cases of atelectasis, two cases of bronchiectasis, two cases of suspected tuberculosis, one case of interstitial pneumonia, one case of atypical mycobacterial infection, one case of suspected eosinophilic pneumonia, and one case of consolidation in chest radiography.

\section{Results}

CYTOLOGY FINDINGS

The cytological features of the bronchoalveolar lavage fluid were similar in all three cases. The smears contained globules with amorphous or granular structures, between 20 and $50 \mu \mathrm{m}$ in diameter, stained green. Some globules were stained green but had an orange zone in the centre. They were found as solitary elements in the smears, with approximately 5-10 per slide. Finely granular or amorphous material was apparent in the background along with scattered neutrophils and lymphocytes. The globules and the amorphous material were PAS positive (fig 1). Focal clusters of alveolar macrophages with foamy or vacuolated cytoplasm were noted.

In the 31 control bronchoalveolar lavage specimens, these globules were not detected. Occasionally, degenerated squamous cells of the same size were observed, at 0-2 per slide, but these could be differentiated by differences
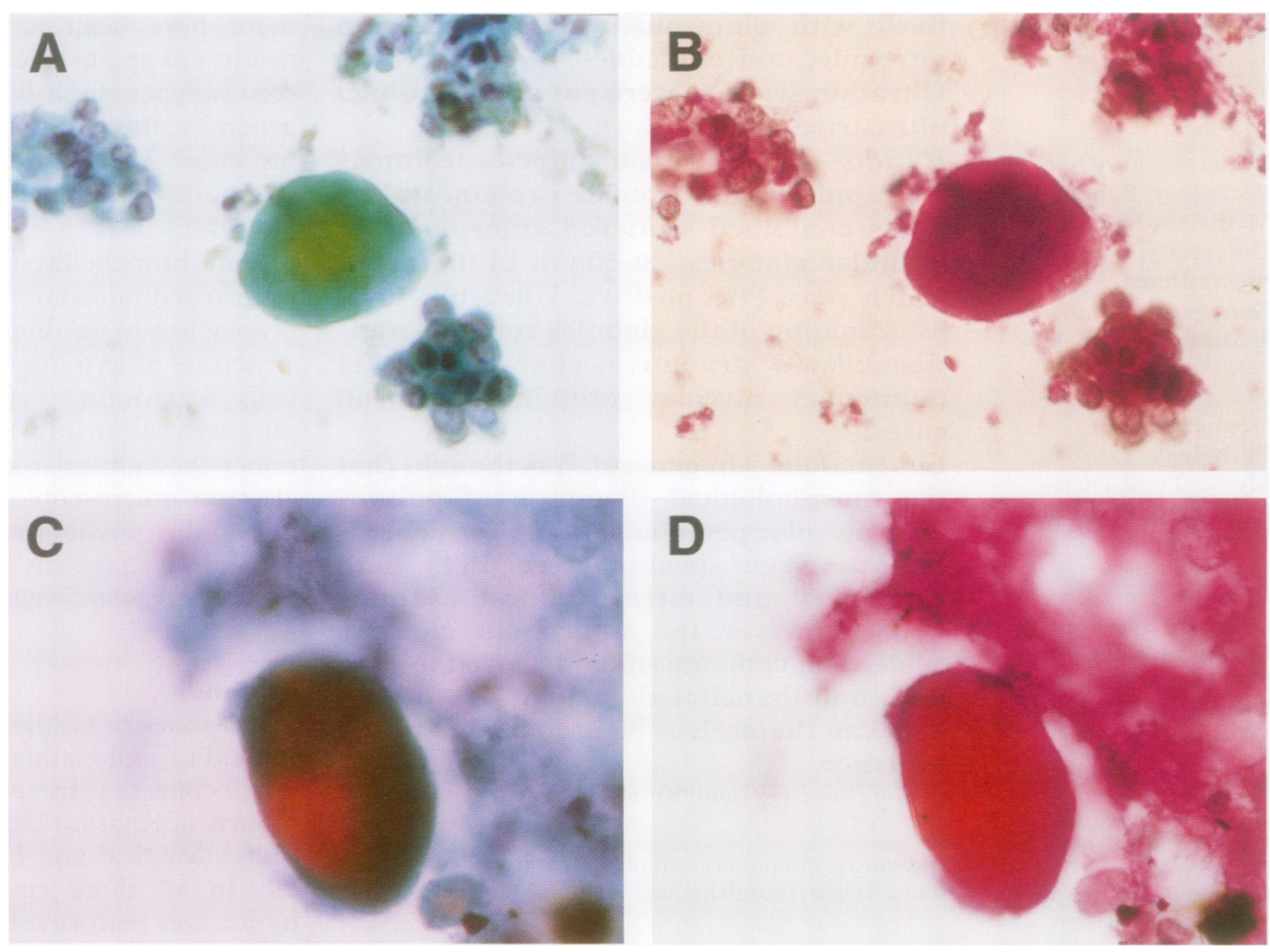

Figure 1 (A) A typical globule detected in a Papanicolaou stained bronchoalveolar lavage smear of case 2; (B) the same globule stained with periodic acid-Schiff (PAS). (C) Another globule stained with Papanicolaou found in case 3; (D) the same globule stained with PAS; note the PAS positive amorphous material in the background. 


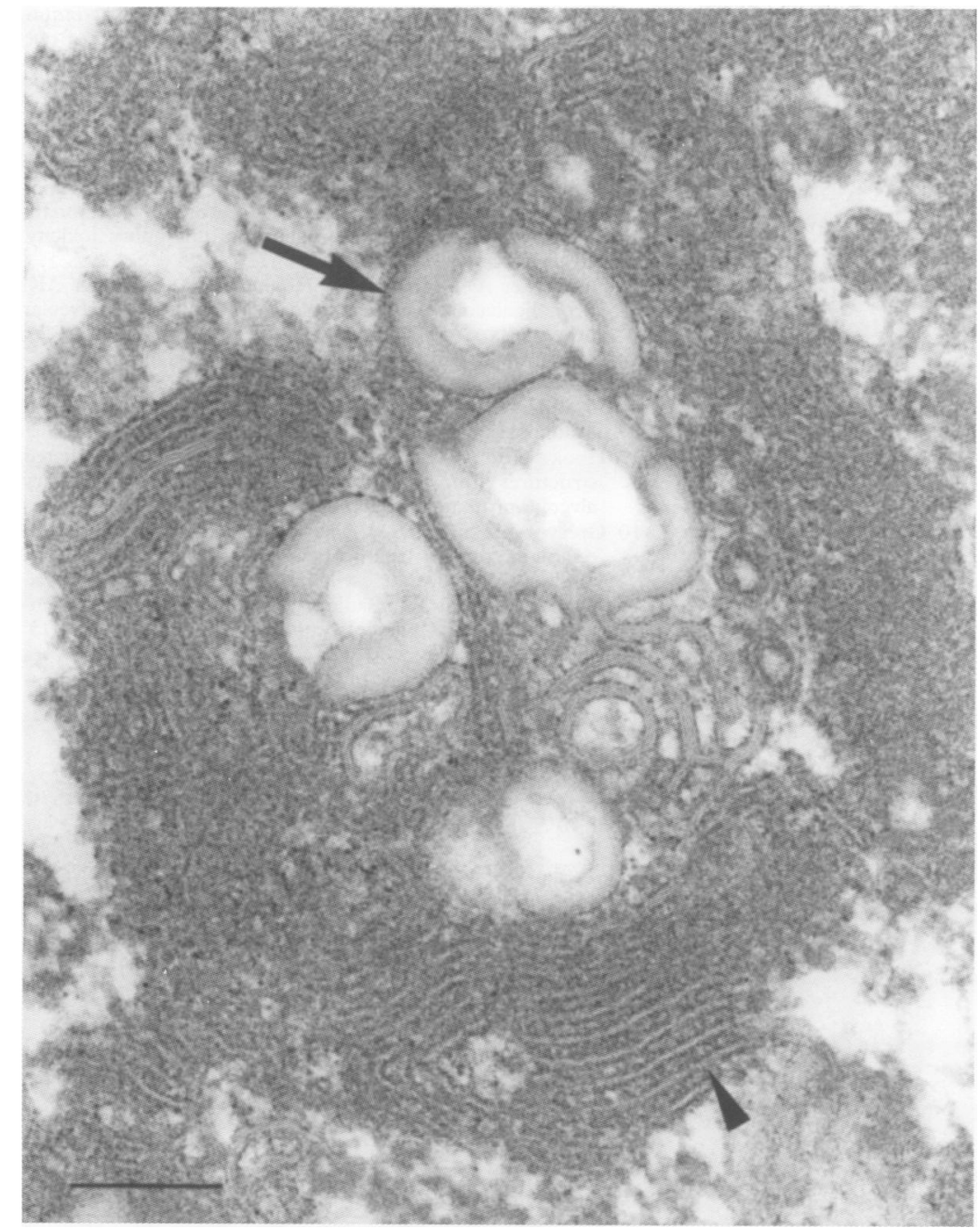

Figure 2 Ultrastructural findings for a globule found in a routine cytology bronchoalveolar lavage smear. The arrow indicates a type B multilamellated structure forming regularly repeated concentric lamellar layers. Its periodicity is in the range of 4-5 $\mathrm{nm}$. The arrowhead indicates a type D multilamellated structure consisting of electron dense double layers alternating with wider electron lucent layers. The double layers measured 8-10 nm in width and the wider layers were $20-30 \mathrm{~nm}$. (Original magnification, $\times 60000 ;$ bar $=200 \mathrm{~nm}$.)

in thickness, the presence of a degenerated nucleus, and the lack of granular structures.

\section{ULTRASTRUCTURAL FINDINGS}

Seven globules detected by cytological examination could be examined ultrastructurally. They were all found to consist of collections of round or oval shaped electron lucent structures, approximately $200-600 \mathrm{~nm}$ in diameter. Although their finer structure was almost destroyed, parts were made up of multilamellated structures consisting of electron dense double layers alternating with wider electron lucent layers. In other areas, concentric multilamellated structures with a periodicity in the 4-5 nm range were found (fig 2). These two different multilamellated structures were compatible with the type $\mathrm{D}$ and type $\mathrm{B}$ multilamellated structures described by Takemura $e t a l,{ }^{8}$ respectively. Type $\mathrm{D}$ multilamellated structures were detected in cases 1 and 3 while type B structures were detected in cases 1 and 2 .

\section{Discussion}

In bronchoalveolar lavage specimens, amorphous or granular globules stained orange or green by Papanicolaou staining have been described to be characteristic of pulmonary alveolar proteinosis. ${ }^{4-7}$ Although ultrastructural findings have been described in several papers, bronchoalveolar lavage sediments fixed with glutaraldehyde or bronchoalveolar lavage cell blocks were used. ${ }^{4-9}$ Therefore, the globules seen in Papanicolaou stained bronchoalveolar lavage smears have hitherto not been linked directly with ultrastructural findings. Furthermore, because in most hospitals cytology materials are fixed routinely with ethanol as smears, ultrastructural examination for diagnosis is not practical.

In this study, however, globules themselves could be examined ultrastructurally, and although most of the ultrastructural features were destroyed as a result of poor alcohol fixation, it was confirmed that the globules comprised collections of multilamellated structures. Takemura et al examined the ultrastructural findings of bronchoalveolar lavage sediments from pulmonary alveolar proteinosis patients that had first been fixed with glutaraldehyde and divided them into four types of multilamellated structures, from $A$ to $D{ }^{8}$ According to their description, type A (the major component) consisted of concentric trilaminar structures that comprised two electron dense layers and a central lucent layer (5.7$7.5 \mathrm{~nm}$ in overall width) alternating with wider (25-30 nm) electron lucent layers. Type B were formed by concentric lamellae with a $5.0-5.3 \mathrm{~nm}$ periodicity. Type C were composed of wavy, electron dense lamellae with a $4.0-4.5 \mathrm{~nm}$ periodicity. Type $\mathrm{D}$ were conglomerated masses of intricately arranged double or triple electron dense layers $(7.5-13.5 \mathrm{~nm}$ wide) alternating with wider $(30-40 \mathrm{~nm})$ electron lucent layers. Comparison with their detailed findings for the four types allowed identification of types B and D in the globules of the bronchoalveolar lavage fluid smears in this study. In other reports using bronchoalveolar lavage sediments or cell blocks, the structures were not categorised into the four types, but from the photographs we can identify the type A multilamellated structure ${ }^{4-79}$ and the type D structure. ${ }^{9}$

In this study, the typical type A multilamellated structure, which is normally the major component, was not detected. It is possible that it is destroyed by routine alcohol fixation and that the electron lucent round or oval structures might be equivalent to type A structures. However, because their inner structures were destroyed, there is no definitive proof. On the other hand, the type B structure was well preserved and the type D multilamellated structure was partly preserved, despite the alcohol fixation. Although the routine alcohol fixation had modified the ultrastructure, it was confirmed that the globules seen in Papanicolaou stained smears comprise multilamellated structures at the electron microscope level.

The important differential diagnosis of pulmonary alveolar proteinosis is from $\mathrm{Pneu}$ mocystis carinii pneumonia. In $P$ carinii pneumonia the bronchoalveolar lavage smear contains characteristic findings described as a "foamy mass" or "foamy exudate". ${ }^{1011} \mathrm{We}$ think that the globules of pulmonary alveolar 
proteinosis can be differentiated from this foamy mass by Papanicolaou staining. When the differentiation is difficult, PAS staining and Grocott methenamine silver staining are useful.

In conclusion, confirmation that the specific PAS positive globular structures found in routinely processed bronchoalveolar lavage fluid smears comprise multilamellated structures indicates that they themselves can be used diagnostically as evidence for pulmonary alveolar proteinosis.

We are grateful to the members of the Electron Microscope Laboratory Center of Kitasato University, School of Medicine for preparing electron micrographs.

Rosen SH, Castleman B, Liebow AA. Pulmonary alveolar proteinosis. N Engl f Med 1958;258:1123-42.

2 Dale DH, Hammer SP Pulmonary pathology, 2nd edn. Berlin: Springer Verlag, 1994:745-51.

3 Hasleton PS. Spencer's pathology of the lung. 5th edn. New York: McGraw-Hill, 1996:773-5.
4 Cardillo MR. Pulmonary alveolar proteinosis-a cytomorphological histochemical and ultrastructural study of one case. Arch Anat Cytol Path 1989;37:259-61.

5 Cordonnier C, Fleury-Feith J, Escudier E, Atassi K, Bernaudin JF. Secondary alveolar proteinosis is a reversible cause of respiratory failure in leukemic patients. $\mathrm{Am} f$ Respir Crit Care Med 1994;149:788-94.

6 Mermolja M, Rott T, Deeljak A. Cytology of bronchoalveolar lavage in some rare pulmonary disorders: pulmonary alveolar proteinosis and amiodarone pulmonary toxicity. Cytopathology 1994;5:9-16.

7 Burkhalter A, Silverman JF, Hopkins MB, Geisinger AK. Bronchoalveolar lavage cytology in pulmonary alveolar proteinosis. Am f Clin Pathol 1996;106:504-10.

8 Takemura T, Fukuda Y, Harrison M, Ferrans VJ. Ultrastructural, histochemical, and freeze-fracture evaluation of multilamellated structures in human pulmonary alveolar proteinosis. Am f Anat 1987;179:258-68.

9 Hook GE, Gilmore LB, Talley FA. Multilamellated structures from the lungs of patients with pulmonary alveolar proteinosis. Lab Invest 1984;50:711-25.

10 Greaves TS, Strigle SM. The recognition of Pneumocysti carinii in routine Papanicolaou-stained smears. Acta Cytol 1985;29:714-20.

11 Ghali VS, Garcia RL, Skolom J. Fluorescence of Pneumocystis carinii in Papanicolaou smears. Hum Pathol 1984;15: 907-9. 